\title{
Computational codon optimization of synthetic gene for protein expression
}

Bevan Kai-Sheng Chung ${ }^{1,2,3}$ and Dong-Yup Lee Le $^{1,2,3^{*}}$

\begin{abstract}
Background: The construction of customized nucleic acid sequences allows us to have greater flexibility in gene design for recombinant protein expression. Among the various parameters considered for such DNA sequence design, individual codon usage (ICU) has been implicated as one of the most crucial factors affecting mRNA translational efficiency. However, previous works have also reported the significant influence of codon pair usage, also known as codon context (CC), on the level of protein expression.

Results: In this study, we have developed novel computational procedures for evaluating the relative importance of optimizing ICU and CC for enhancing protein expression. By formulating appropriate mathematical expressions to quantify the ICU and CC fitness of a coding sequence, optimization procedures based on genetic algorithm were employed to maximize its ICU and/or CC fitness. Surprisingly, the in silico validation of the resultant optimized DNA sequences for Escherichia coli, Lactococcus lactis, Pichia pastoris and Saccharomyces cerevisiae suggests that CC is a more relevant design criterion than the commonly considered ICU.

Conclusions: The proposed CC optimization framework can complement and enhance the capabilities of current gene design tools, with potential applications to heterologous protein production and even vaccine development in synthetic biotechnology.
\end{abstract}

\section{Background}

Recent developments in artificial gene synthesis have enabled the construction of synthetic gene circuits [1] and even the synthesis of whole bacterial genome [2]. The introduction of synthetic genes into a living system can either modulate existing biological functions or give rise to novel cellular behavior. In this sense, de novo gene synthesis is a valuable synthetic biological tool for biotechnological studies, which typically aims to improve tolerance to toxic molecules, retrofit existing biosynthetic pathways, design novel biosynthetic pathways and/or enhance heterologous protein production $[3,4]$. In the aspect of recombinant protein production, natural genes found in wild-type organisms are usually transformed into the heterologous hosts for recombinant expression. This approach typically results in poorly

\footnotetext{
* Correspondence: cheld@nus.edu.sg

'Department of Chemical and Biomolecular Engineering, National University of Singapore, 4 Engineering Drive 4, Singapore 117576, Singapore

${ }^{2}$ NUS Graduate School for Integrative Sciences and Engineering, National University of Singapore, 28 Medical Drive, \#05-01, Singapore 117456, Singapore

Full list of author information is available at the end of the article
}

expressed recombinant protein since the wild-type foreign genes have not been evolved for optimum expression in the host. Thus, it is highly desirable to harness the flexibility in synthetic biology to create customized artificial gene designs that are optimal for heterologous protein expression. To aid the gene design process, computational tools have been developed for designing coding sequences based on some performance criteria.

Specifically, the degeneracy of the genetic code, reflected by the use of sixty-four codons to encode twenty amino acids and translation termination signal, leads to the situation whereby all amino acids, except methionine and tryptophan, can be encoded by two to six synonymous codons. Notably, the synonymous codons are not equally utilized to encode the amino acids, thus resulting in phenomenon of codon usage bias which was first reported in a study that examines the frequencies of 61 amino acid codons (i.e. termination codons are excluded) in 90 genes [5]. The emergence of codon usage bias in organisms has been largely attributed to natural selection, mutation, and genetic drift [6]. More importantly, codon usage bias has been shown to be correlated to gene expression level $[7,8]$. As a result, this bias 
has been proposed as an important design parameter for enhancing recombinant protein production in heterologous expression hosts [9]. Consequently, the algorithms implemented in many of the sequence design software tools, such as Codon optimizer [10], Gene Designer [11], and OPTIMIZER [12], are mainly focused on the frequency of individual codon occurrences. Notably, the popular web-based software, known as the Java Codon Adaptation Tool (JCat), is integrated with the PRODORIC database to allow convenient retrieval of prokaryotic genetic information [13,14]. However, apart from individual codon usage (ICU) bias, nonrandom utilization of adjacent codon pairs in organisms has also been reported in several studies $[15,16]$. This phenomenon is termed "codon context" as it implicates some "rule" for organizing neighboring codons as a result of potential tRNA-tRNA steric interaction within the ribosomes $[17,18]$. Codon context (CC) was shown to correlate with translation elongation rate such that the usage of rare codon pairs decreased protein translation rates [19]. Therefore, the incorporation of $\mathrm{CC}$ has been proposed in the conventional ICU-based gene optimization algorithm GeneOptimizer [20]. Furthermore, a patented technology, known as "Translation Engineering", demonstrated that better enhancement in translational efficiency is achievable by optimizing codon pair usage in addition to ICU optimization [21]. However, there is yet a study to investigate the relative effects of ICU and CC on protein expression. To address this issue, we propose a computational analysis to evaluate the performance of sequences generated by various ICU and CC optimization approaches.

In this study, we applied novel computational procedures to generate DNA sequences exhibiting optimal ICU and CC in Escherichia coli, Lactococcus lactis, Pichia pastoris and Saccharomyces cerevisiae based on information obtained from omics data analysis. While E. coli and $S$. cerevisiae has been model organisms for recombinant protein production studies, we also consider codon optimization in the Gram-positive bacterium L. lactis and methylotrophic yeast $P$. pastoris since they are also promising candidates for expressing recombinant proteins $[22,23]$. Assuming that the native DNA sequences of highly expressed genes have evolved to exhibit optimal ICU and CC for high in vivo expression, we demonstrated the efficacy of our computational approaches by performing a leave-one-out cross-validation on the highexpression genes for each expression host.

\section{Results}

\section{Codon optimization formulation}

To investigate the relative importance of ICU and CC towards designing sequences for high protein expression, we implemented three computational procedures: the individual codon usage optimization (ICO) method generates a sequence with optimal ICU only; the codon context optimization (CCO) method optimizes sequences with regard to codon context only; and the multiobjective codon optimization (MOCO) method simultaneously considers both ICU and CC. Thus, the resultant sequence is ICU-/CC-optimal when its ICU/CC distribution is closest to the organism's reference ICU/ $\mathrm{CC}$ distribution calculated based on the sequences of native high-expression genes. Based on the mathematical formulation presented in Methods, the ICO problem can be described as the maximization of ICU fitness, $\Psi_{\mathrm{ICU}}$ (see Eqn. 23), subject to the constraint that the codon sequence can be translated into the target protein (see Eqns. 3, 4 and 11). Due to the discrete codon variables and nonlinear fitness expression of $\Psi_{\mathrm{ICU}}$, ICO is classified a mixed-integer nonlinear programming (MINLP) problem. Nonetheless, it can be linearized using a strategy shown in an earlier study by decomposing the nonlinear $\left|p_{0}^{k}-p_{1}^{k}\right|$ term (see Equation 23) into a series of linear and integer constraints which consist of binary and positive real variables [24]. The resultant mixedinteger linear programming (MILP) problem can be solved using well established computational methods such as either branch-and-bound and branch-and-cut [25]. However, due to the large and discrete search space which contains all possible DNA sequences that can encode the target protein, solving the MILP using these methods may require a long computational time. Thus, alternative methods, such as GASCO [26] and QPSOBT [27], have been proposed for solving ICO using genetic algorithm and particle swarm optimization. Although these heuristic methods are more efficient than conventional MILP solving procedures, they still require a significant amount of computational resources due to the iterative nature of the algorithms. To circumvent the high computational costs, we developed the non-iterative method for solving ICO using the following steps:

I1. Calculate the host's individual codon usage distribution, $p_{0}^{k}$.

I2. Calculate the subject's amino acid counts, $\theta_{\mathrm{AA}, 1}^{j}$.

I3. Calculate the optimal codon counts for the subject using the expression: $\theta_{\mathrm{C}, \mathrm{opt}}^{\mathrm{k}}=p_{0}^{\mathrm{k}} \times \sum_{j=1}^{21}\left[\theta_{\mathrm{A}, 1}^{\mathrm{j}} \times 1\left\{\alpha^{\mathrm{j}}=\right.\right.$ $\left.\left.f\left(\kappa^{\mathrm{k}}\right)\right\}\right] \quad \forall \mathrm{k} \in\{1,2, \ldots, 64\}$.

I4. For each $\tau_{i}$ in the subject's sequence, randomly assign a codon $\kappa^{k}$ if $\theta_{\mathrm{C}}^{k}>0$, and decrement $\theta_{\mathrm{C}, \mathrm{opt}}^{k}$ by one.

I5. Repeat step I4 for all amino acids of the target protein from $\tau_{1,1}$ to $\tau_{n, 1}$.

Similarly, CCO can be formulated as the maximization of CC fitness, $\Psi_{\mathrm{CC}}$ (see Eqn. 26), subject to the constraint 
that the codon pair sequence can be translated into the target protein (see Eqns. 7, 8 and 12). To find the solution for $\mathrm{CCO}$, the procedure in ICO may not be applicable due to the computational complexity which arises from the dependency of adjacent codon pairs. For example, given a codon pair "AUG-AGA" in a 5'-3' direction, the following codon pair must only start with "AGA". Therefore, if we had adopted the ICO procedure to directly identify the codon pairs and randomly assign them to the respective amino acid pairs, there could be conflicting codon pair assignments in certain parts of the sequence. Since the characteristic of independency, which was exploited to develop a simple solution procedure for ICO, is absent in the CCO problem, we resort to a more sophisticated computational approach.

The CCO problem can be conceptualized in a similar way as the well-known traveling salesman problem whereby the traversing from one codon to the next adjacent codon is analogous to the salesman traveling from one city to the next [28]. Since there will be a "cost" incurred by taking a particular "codon path", the CCO problem aims to minimize of the total cost for traveling a codon path that is able to code the desired protein sequence. However, the CCO problem is more complex than the traveling salesman problem due to the nonlinear cost function evaluated based on the frequency of codon pair occurrence (see Materials and Methods). For an average sized protein consisting 300 amino acids, the total number of codon paths can be as many as $10^{100}$. Finding an optimal solution for such a large-scale combinatorial problem within an acceptable period of computation time can only be achieved via heuristic optimization methods. Incidentally, the use of genetic algorithm [29] provides an intuitive framework whereby codon path candidates are "evolved" towards optimal CC through techniques mimicking natural evolutionary processes such as selection, crossover or recombination and mutation. Thus, the procedure for solving $\mathrm{CCO}$ is as follows:

\section{C1. Randomly initialize a population of coding} sequences for target protein.

C2. Evaluate the CC fitness of each sequence in the population.

C3. Rank the sequences by CC fitness and check termination criterion.

C4. If termination criterion is not satisfied, select the "fittest" sequences (top 50\% of the population) as the parents for creation of offsprings via recombination and mutation.

C5. Combine the parents and offsprings to form a new population.

C6. Repeat steps $\mathrm{C} 2$ to $\mathrm{C} 5$ until termination criterion is satisfied.
In step C3, the termination criterion depends on the degree of improvement in best CC fitness values for consecutive generations of the genetic algorithm. If the improvement in CC fitness across many generations is not significant, the algorithm is said to have converged. In this study, the CC optimization algorithm is set to terminate when there is less than $0.5 \%$ increase in CC fitness across 100 generations, i.e. $\Psi_{\mathrm{CC}}^{(r+100)} / \Psi_{\mathrm{CC}}^{(r)}<0.005$ where $r$ refers to the $r^{\text {th }}$ generation of the genetic algorithm. When the termination criterion is not satisfied, the subsequent step C4 will perform an elitist selection such that the fittest $50 \%$ of the population are always selected for reproduction of offsprings through recombination and mutation. During recombination, a pair of parents is chosen at random and a crossover is carried out at a randomly selected position in the parents' sequences to create 2 new individuals as offsprings. The offsprings subsequently undergo a random point mutation before they are combined with the parents to form the new generation.

Unlike traditional implementations of genetic algorithm where individuals in the population are represented as as 0-1 bit strings, the presented CC optimization algorithm represents each individual as a sequential list of character triplets indicating the respective codons. Therefore, the codons can be manipulated directly with reference to a hash table which defines the synonymous codons for each amino acid. As a result, the protein encoded by the coding sequences is always the same in the genetic algorithm since crossovers only occur at the boundary of the codon triplets and mutation is always performed with reference to the hash table of synonymous codons for each respective amino acid.

Based on the formulations for ICU and CC optimization, the MOCO problem, which is an integration of both, can be described as maximizing both ICU and CC fitness, i.e. $\max \left(\Psi_{\mathrm{ICU}}, \Psi_{\mathrm{CC}}\right)$, subject to the constraints that both the codon and codon pair sequences can be translated into the target protein sequence. As such, due to the complexity attributed to $\mathrm{CC}$ optimization, solution to $\mathrm{MOCO}$ will also require a heuristic method. In this case, the nondominated sorting genetic algorithm-II (NSGA-II) is used to solve the multi-objective optimization problem [30]. The procedure for NSGA-II is similar to that presented for CC optimization except for additional steps required to identify the nondominated solution sets and the ranking of these sets to identify the pareto optimum front. The NSGA-II procedure for solving the $\mathrm{MOCO}$ problem is as follows:

M1. Randomly initialize a population of coding sequences for target protein.

M2. Evaluate ICU and CC fitness of each sequence in the population.

M3. Group the sequences into nondominated sets and rank the sets. 
M4. Check termination criterion.

M5. If termination criterion is not satisfied, select the "fittest" sequences (top 50\% of the population) as the parents for creation of offsprings via recombination and mutation.

M6. Combine the parents and offsprings to form a new population.

M7. Repeat steps M2 to M5 until termination criterion is satisfied.

The identification and ranking of nondominated sets in step M3 is performed via pair-wise comparison of the sequences' ICU and CC fitness. For a given pair of sequences with fitness values expressed as $\left(\Psi_{\mathrm{ICU}}^{1}, \Psi_{\mathrm{CC}}^{1}\right)$ and $\left(\Psi_{\mathrm{ICU}}^{2}, \Psi_{\mathrm{CC}}^{2}\right)$, the domination status can be evaluated using the following rules:

- If $\left(\Psi_{\mathrm{ICU}}^{1}>\Psi_{\mathrm{ICU}}^{2}\right)$ and $\left(\Psi_{\mathrm{CC}}^{1} \geq \Psi_{\mathrm{CC}}^{2}\right)$, sequence 1 dominates sequence 2 .
- If ( $\left.\Psi_{\mathrm{ICU}}^{1} \geq \Psi_{\mathrm{ICU}}^{2}\right)$ and $\left(\Psi_{\mathrm{CC}}^{1}>\Psi_{\mathrm{CC}}^{2}\right)$, sequence 1 dominates sequence 2 .

- If $\left(\Psi_{\mathrm{ICU}}^{1}<\Psi_{\mathrm{ICU}}^{2}\right)$ and $\left(\Psi_{\mathrm{CC}}^{1} \leq \Psi_{\mathrm{CC}}^{2}\right)$, sequence 2 dominates sequence 1 .

- If $\left(\Psi_{\mathrm{ICU}}^{1} \leq \Psi_{\mathrm{ICU}}^{2}\right)$ and $\left(\Psi_{\mathrm{CC}}^{1}<\Psi_{\mathrm{CC}}^{2}\right)$, sequence 2 dominates sequence 1 .

Whenever a particular sequence is found to be dominated by another sequence, the domination rank of the former sequence is lowered. As such, the grouping and sorting of the nondominated sets are performed simultaneously in step M3 (Figure 1). In the original nondominated sorting algorithm [30], the set of individuals that is dominated by every individual is stored in memory. Therefore, for a total population of $n$, the total storage requirement is $O\left(n^{2}\right)$. However, for the abovementioned algorithm, only $O(n)$ storage is required for storing the domination value of each individual. In terms of computational complexity, both the original and modified algorithm requires at most

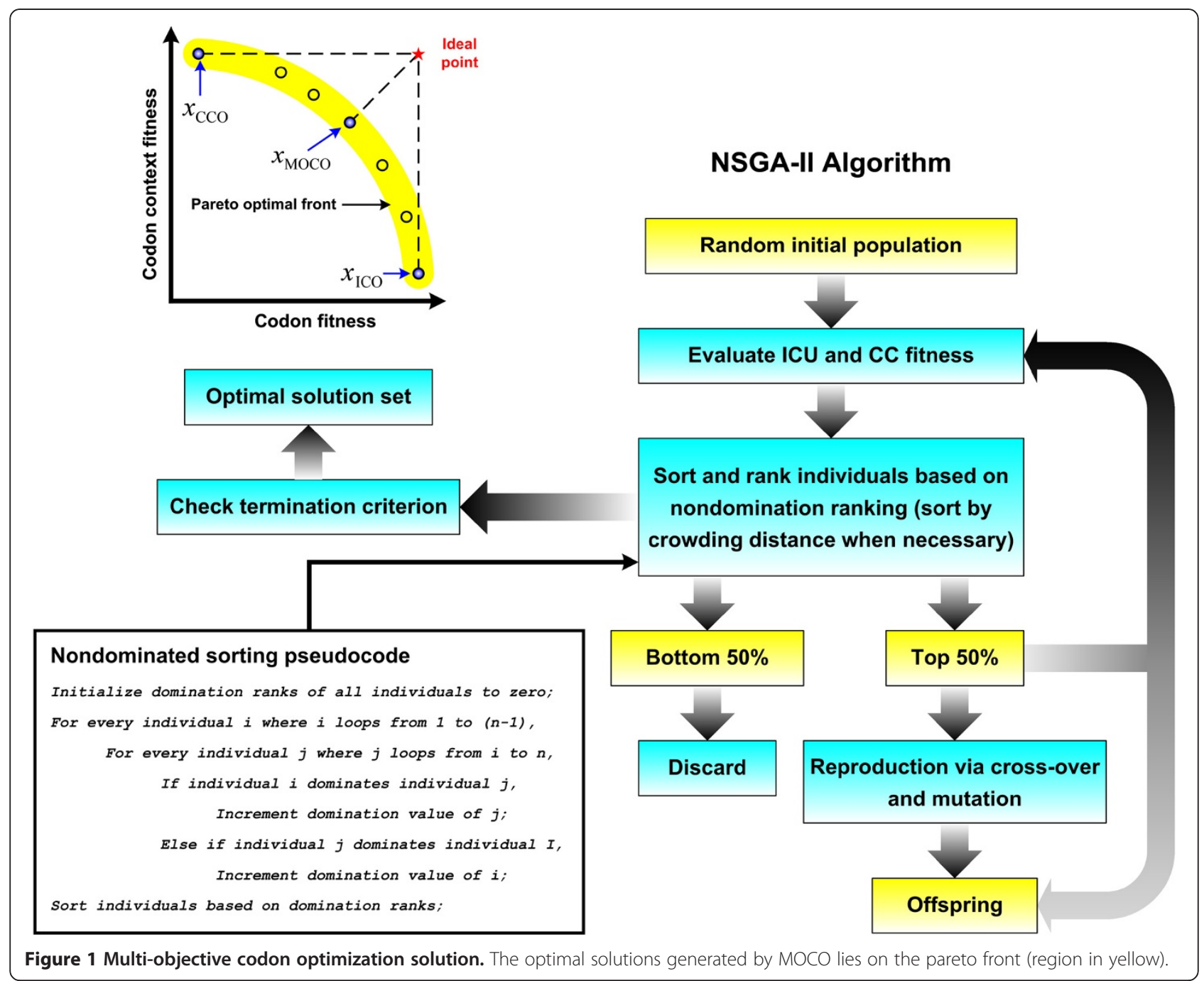


$O\left(m n^{2}\right)$ computations for $m$ objective values since all the $n$ individuals have to be compared pair-wise for every objective to be optimized. Therefore, the nondominated sorting algorithm presented in this thesis is superior on the whole, especially with regards to computational storage requirement which can become an important issue when dealing with long coding sequences.

The output of multi-objective optimization is a set of solutions also known as the pareto optimal front. Since the aim of MOCO is to examine the relative effects of ICU and CC optimization, it is not necessary to analyze all the sequences in the pareto optimal front. Instead, the solution which is nearest to the ideal point will represent the sequence with balanced ICU and CC optimality. As such, the solutions of ICO, CCO and MOCO will subsequently be referred to as $x_{\mathrm{ICO}}, x_{\mathrm{CCO}}$ and $x_{\mathrm{MOCO}}$ respectively (Figure 1). The program for performing ICO, CCO and MOCO can be downloaded from the following link: http:// bioinfo.bti.a-star.edu.sg/tool/CodonOptimization/.

\section{Finding the codon preference}

The entire workflow for codon optimization of a target protein sequence begins with the identification of the host's preferred ICU and CC distributions as the reference (Figure 2). These ICU and CC distributions should ideally capture codon usage patterns that correspond to efficient translation of mRNA to protein. Therefore, the first step of codon optimization identifies the reference ICU and CC distributions by characterizing the underlying mechanisms of efficient translation which can be achieved through transcriptome, translatome and proteome profiling as demonstrated in earlier studies [31,32]. However, such large-scale experimental data are not readily available for the extraction of codon usage preference information in all the expression hosts considered in this study. Alternatively, it is assumed that the organisms have evolved to conserve resources by producing high amounts of transcripts for genes that will also be efficiently translated. As such, the widely available transcriptome data from microarray experiments can be used to identify the highly expressed and efficiently translated genes. Thus, the codon pattern of the host's native high-expression genes will be a suitable reference point for codon optimization.

The step for selecting high-expression genes codon pattern for codon optimization is only relevant if the following two conditions are true: (1) ICU and CC distributions of high-expression genes are significantly biased and nonrandom; and (2) there is a significant difference in ICU and CC distribution between highly expressed genes and all the genes in the host organism's genome. It is noted that if the first condition is false, there is no codon (pair) bias and codons can be assigned randomly based on a uniform distribution; if the second condition is false, the computation of ICU and CC distributions based on all the genes in the genome will be sufficient to characterize the ICU and CC preference of the organism without the need for selecting high-expression genes.

To determine the significance of ICU and CC biases, we applied the Pearson's chi-squared test (see Materials and Methods). Using a p-value cut-off of 0.05 , the ICU and CC distributions of at least $80 \%$ of the amino acids (pairs) amenable to the chi-squared test were found to be significantly biased in the micro-organisms (Table 1). In the high-expression genes, aspartate was found to be the only one among all amino acids exhibiting an ICU distribution that is not significantly different from the unbiased distribution for E. coli, P. pastoris and S. cerevisiae. Similarly, more than $80 \%$ of the amino acids (pairs) show significant difference in ICU and CC distributions between high-expression genes and all genes in the genomes of these three microbes. Contrastingly, $80 \%$ amino acids did not show significant difference in CC distributions between high-expression genes and all genes in $L$. lactis, suggesting that the selection of highly expressed genes may not be required to establish the $\mathrm{CC}$ preference of $L$. lactis. By applying the principal component analysis, we can observe that the ICU and CC distributions for all types of genes in L. lactis are close to one another when compared to genes from other organisms (Figure 3). This indicates that the short listing of highly expressed genes may not be necessary for organisms like L. lactis. Nonetheless, we recommend the identification of high-expression genes to characterize the ICU and CC preference of any host, such that there is a better level of confidence that the optimized recombinant gene can be efficiently expressed.

\section{Performance of codon optimization methods}

The performance of each optimization approach was evaluated using a leave-one-out cross-validation, where a gene is randomly selected from the entire set of highexpression genes for sequence optimization while the rest of the genes will be used as the training set to calculate the reference ICU and CC distribution (Figure 4). The predicted optimum sequences are compared with the original native sequences to evaluate the performance of each codon optimization approach (see Additional file 1 for the sequences of the wild-type and optimized genes). As the degree of similarity to the wild-type high expression genes indicates the gene expressivity potential of the optimized sequences, the quality of each optimized sequence was measured in terms of the percentage of codons matching the corresponding native sequence, denoted by $P_{M}$. From the results, the $x_{\mathrm{ICO}}, x_{\mathrm{CCO}}$ and $x_{\mathrm{MOCO}}$ solutions were generally found to be more similar to the native genes than the random sequences generated by RCA indicating that all the optimization approaches are indeed capable of 


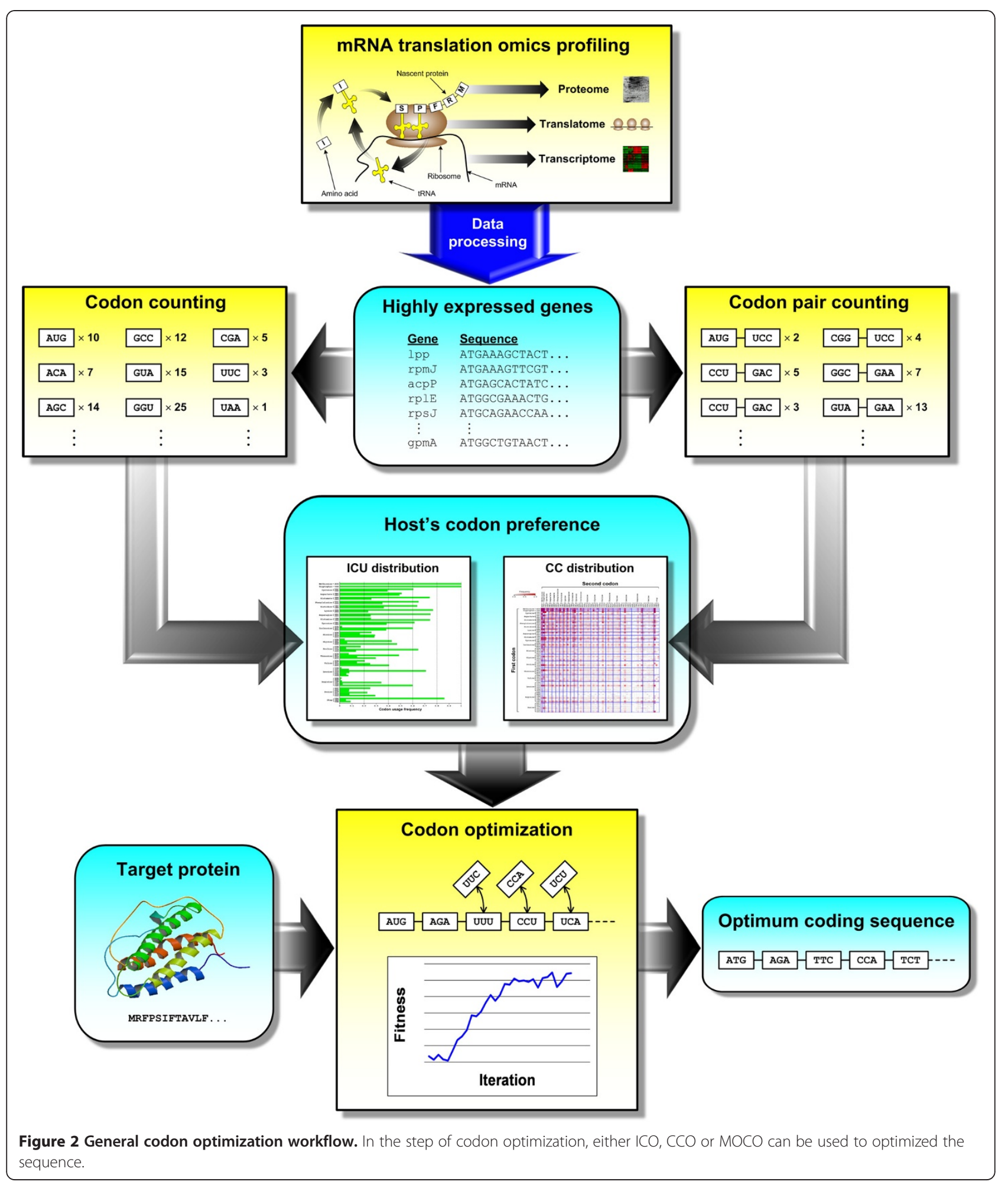

improving the codon usage pattern compared to the control (Figure 4). The $P_{M}$ values of $x_{\mathrm{ICO}}, x_{\mathrm{CCO}}, x_{\mathrm{MOCO}}$ and $x_{\mathrm{RCA}}$ sequences for each gene are further compared in a "tournament" style to show the relative performance of each optimization method. In the tournament matrix
(Table 2), each cell shows the number of wins by the method in the left-most column against that in the uppermost row. Whenever the numbers of wins and losses (i.e. cells diagonally opposite of each other) do not sum up to 100 , the shortfall will be equal to the number of draws. 
Table 1 ICU and CC biasness analysis

\begin{tabular}{|c|c|c|c|c|c|c|c|c|}
\hline \multirow[b]{2}{*}{ Null hypothesis $\left(H_{0}\right)$} & \multicolumn{2}{|c|}{ E. coli } & \multicolumn{2}{|c|}{ L. lactis } & \multicolumn{2}{|c|}{ P. pastoris } & \multicolumn{2}{|c|}{ S. cerevisiae } \\
\hline & $D^{H}=U$ & $D^{H}=D^{A}$ & $D^{H}=U$ & $D^{H}=D^{A}$ & $D^{H}=U$ & $D^{H}=D^{A}$ & $D^{H}=U$ & $D^{H}=D^{A}$ \\
\hline Alternative hypothesis $\left(H_{1}\right)$ & $D^{H} \neq U$ & $D^{H} \neq D^{A}$ & $D^{H} \neq U$ & $D^{H} \neq D^{A}$ & $D^{H} \neq U$ & $D^{H} \neq D^{A}$ & $D^{H} \neq U$ & $D^{H} \neq D^{A}$ \\
\hline No. of biased amino acids (P-value $<0.05$ ) & 18 & 17 & 19 & 17 & 18 & 19 & 18 & 19 \\
\hline No. of unbiased amino acids (P-value $\geq 0.05$ ) & 1 & 2 & 0 & 2 & 1 & 0 & 1 & 0 \\
\hline No. of singular amino acids & 2 & 2 & 2 & 2 & 2 & 2 & 2 & 2 \\
\hline No. of unevaluated amino acids (Expect count $<5$ ) & 0 & 0 & 0 & 0 & 0 & 0 & 0 & 0 \\
\hline Total no. of amino acids & 21 & 21 & 21 & 21 & 21 & 21 & 21 & 21 \\
\hline No. of biased amino acid pairs (P-value $<0.05$ ) & 314 & 99 & 327 & 15 & 354 & 259 & 372 & 282 \\
\hline No. of unbiased amino acid pairs (P-value $\geq 0.05$ ) & 26 & 23 & 12 & 65 & 38 & 36 & 19 & 9 \\
\hline No. of singular amino acid pairs & 4 & 4 & 4 & 4 & 4 & 4 & 4 & 4 \\
\hline No. of unevaluated amino acid pairs (Expect count $<5$ ) & 76 & 294 & 77 & 336 & 24 & 121 & 25 & 125 \\
\hline Total no. of amino acid pairs & 420 & 420 & 420 & 420 & 420 & 420 & 420 & 420 \\
\hline
\end{tabular}

Through the comparison of ICO and CCO, the $x_{\mathrm{CCO}}$ solutions have a higher average percentage of codon matches than $x_{\text {ICO }}$ sequences for all four microbes (Figure 4), with at least $90 \%$ of the $x_{\mathrm{CCO}}$ sequences matching the native corresponding sequences better than those generated by ICO (Table 2). This result indicates that CC fitness can be a more important design parameter for sequence optimization than ICU fitness which has been a conventional design criterion implemented in several software tools. While it appears likely that the integration of $\mathrm{CCO}$ with ICO under a multiobjective optimization framework can potentially lead to even better sequence design, results from our MOCO analysis suggest otherwise. The average of $P_{M}$ value of $x_{\mathrm{MOCO}}$ were observed to be lower than that of $x_{\mathrm{CCO}}$, indicating that the consideration of ICU fitness in addition to $\mathrm{CC}$ fitness can be detrimental to the sequence design. To our best knowledge, no such formal evaluation of the relative impact of ICU and CC fitness on synthetic gene design has been presented to date. Hence, based on the promising in silico validation results which implicate $C \mathrm{C}$ as an important design parameter for optimizing sequences, the newly developed CCO procedure can potentially supersede the ICU optimization techniques currently implemented in gene design software tools. It is noted that similar observations on the relative performance of ICO, CCO and MOCO were made when we performed the in silico leave-one-out cross-validation on the set of 27 highexpression genes of $E$. coli reported in an earlier study [33]. Details of this analysis can be found in Additional file 2 .
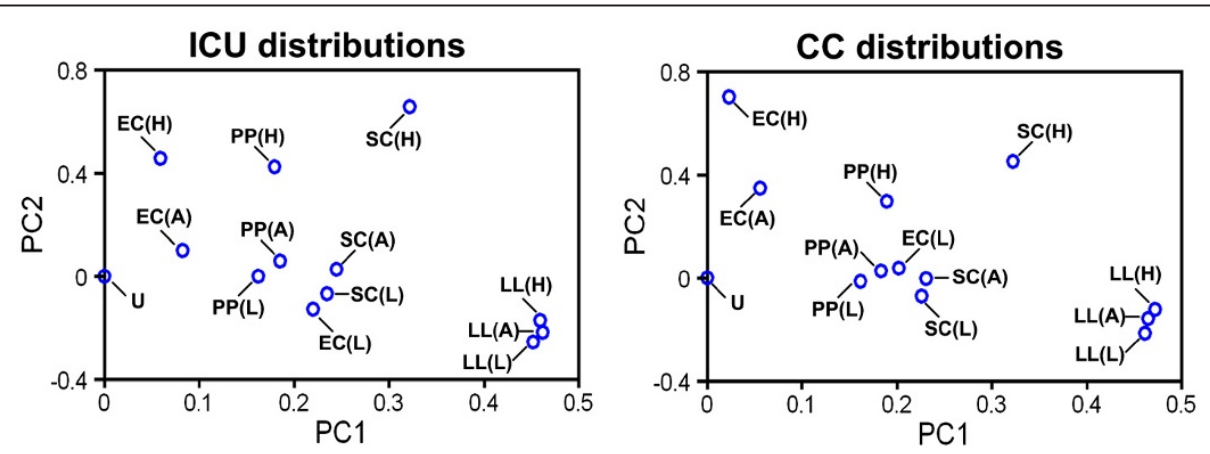

Figure 3 PCA of ICU and CC distributions. The first and second principal components (PC1 and PC2) are plotted to show the differences in the ICU and CC distributions of (top 5\%) high-expression genes (H), (bottom 5\%) low-expression genes (L) and all genes (A) found in the genomes of E. coli (EC), L. lactis (LL), P. pastoris (PP) and S. cerevisiae (SC). The unbiased distribution (U) is also included for each plot as reference. 


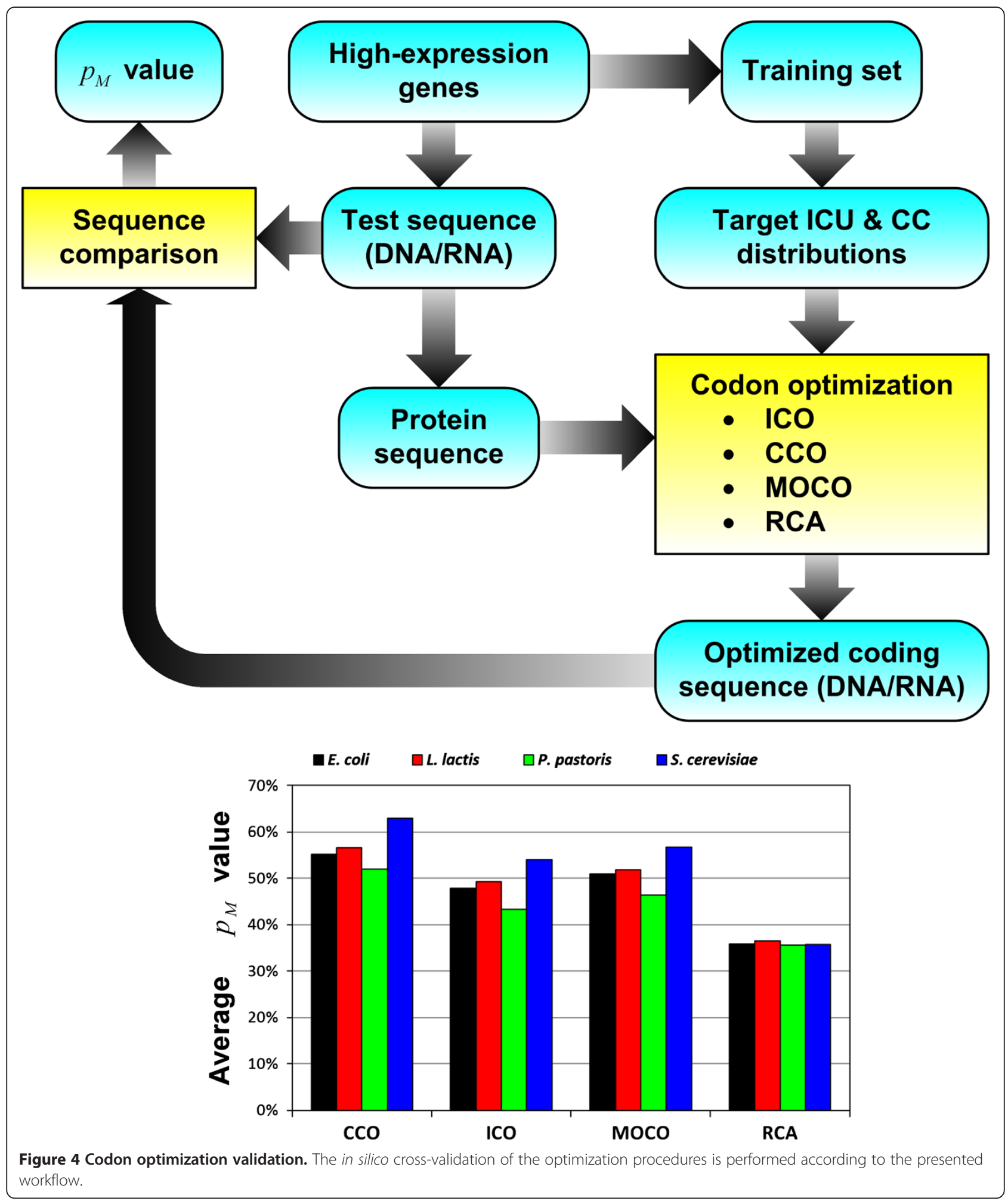

\section{Discussion}

Capturing the preferred codon usage patterns

Earlier codon optimization studies have recommended the usage of high expression genes to design the recombinant gene for efficient heterologous expression [12,13,34]. In the analysis of codon usage patterns, the significant distinction in the ICU and CC distributions between highly expressed and other genes corroborated the relevance of 
Table 2 Tournament matrix

\begin{tabular}{|c|c|c|c|c|}
\hline & $x_{\text {ICO }}$ & $x_{\mathrm{CCO}}$ & $x_{\text {Moco }}$ & $x_{\mathrm{RCA}}$ \\
\hline & & 7 & 19 & 95 \\
\hline & & 2 & 18 & 99 \\
\hline \multirow[t]{4}{*}{$x_{1 C O}$} & & 4 & 15 & 93 \\
\hline & & 5 & 22 & 99 \\
\hline & 92 & & 82 & 97 \\
\hline & 96 & & 93 & 100 \\
\hline \multirow[t]{4}{*}{$x_{C C O}$} & 96 & & 86 & 100 \\
\hline & 93 & & 89 & 99 \\
\hline & 78 & 15 & & 97 \\
\hline & 74 & 5 & & 100 \\
\hline \multirow[t]{4}{*}{$x_{\mathrm{MOCO}}$} & 83 & 12 & & 99 \\
\hline & 75 & 9 & & 99 \\
\hline & 5 & 2 & 3 & \\
\hline & 0 & 0 & 0 & \\
\hline \multirow[t]{2}{*}{$x_{R C A}$} & 6 & 0 & 1 & \\
\hline & 1 & 0 & 0 & \\
\hline
\end{tabular}

For every gene, the $p_{M}$ of the optimal sequences generated by respective optimization approaches are compared pair-wise for each expression host. The numbers of tournament wins/losses by each approach for all the genes in each expression host are added up. The sequences generated by ICO, CCO, $\mathrm{MOCO}$ and RCA are indicated as $x_{1 C O}, x_{C C O}, x_{M O C O}$ and $x_{\mathrm{RCA}}$ respectively. In each cell, the numbers from top-most to bottom-most corresponds to the data for E. coli, L. lactis, P. pastoris and S. cerevisiae, respectively.

identifying high-expression genes to characterize the preferred codon usage patterns. It is noted that although there is codon usage information readily available in the Codon Usage Database (http://www.kazusa.or.jp/codon/) [35], these data may not be useful as prior filtering of highly expressed genes was not performed. Such codon usage data may reflect some degree of preference for "rare" codons, thus leading to low gene expression [36].

Several options are available for quantifying the codon usage patterns. In this study, we have adopted the method of treating the ICU and CC distributions as a vector of frequency values to capture the relative abundance of individual codons and codon pairs. An earlier well-known method for quantifying codon usage bias is the codon adaptation index (CAI). The CAI has been widely used for codon optimization due to its observed correlation with gene expressivity [34]. However, by designing a gene through the maximization of CAI, the resultant coding sequence will become a "one amino acid - one codon" design where $\mathrm{CAI}=1.0$. This sequence design may not be desirable as the overexpression of this gene can lead to very rapid depletion of the specific cognate tRNAs resulting in tRNA pool imbalance, which can in turn cause an increase in translational errors [37]. In this aspect, the ICU fitness measure will be a better performance criterion than CAI since the former allows a small number of rare codons to be included in the final sequence. Furthermore, the calculation of CAI, as described in its original paper [34], is intrinsically based on individual codon usage and does not have the capability to account for codon pairing. Therefore, the information captured by the CC fitness cannot be reflected in the CAI value.

Therefore, the proposed approach of optimizing codons according to the complete ICU and CC distributions of highly expressed genes will be suitable to alleviate the problem of tRNA pool imbalance when the cell is induced to overexpress the target gene. As such, the concept of CAI was not considered in this study as this single value does not capture the details in ICU and CC distributions.

\section{Other potential issues in efficacy of CCO}

Codon usage has been shown to affect the accuracy and speed of translation $[38,39]$. Hence, the concept of CCO implementation is to identify favorable codon pairings that can lead to more efficient protein synthesis process. Notably, an optimization framework based on the dynamic modeling of protein translation has been recently developed to identify suitable codon placements to improve translation elongation speed [40]. Although this method provides a mechanistic understanding of how codon choice affects translation efficiency, it requires a protein translation kinetic model and codon-specific elongation rates which may not be readily available for organisms other than E. coli as shown in previous studies $[32,41]$. Therefore, CCO may be a better alternative as it can achieve the aim of enhancing translation efficiency while having the advantage of utilizing information, including genome sequence and gene expression data, which are easily accessible in public databases such as the Gene Expression Omnibus (www.ncbi.nlm.nih. gov/geo/) and GenBank (www.ncbi.nlm.nih.gov/genbank/). Incidentally, there was evidence suggesting that translation initiation rather than elongation is the rate limiting step [42]. Nonetheless, CCO generated sequences can indirectly increase translation initiation by freeing up more ribosomes through enhanced translation elongation rates. The increased pool of free ribosomes can then help to improve translation initiation by mass action effect.

On the other hand, translation initiation can also be affected by the mRNA structure of the initiation site. At the primary structure level, Shine-Dalgarno sequence and Kozak sequence should be added to the 5 ' end of the coding sequence since previous studies have shown that they are required for recognition of the AUG start codon to initiate translation in prokaryotes and eukaryotes, respectively, [43]. At the secondary structure level, it was found that hairpin, stem-loop and pseudoknot mRNA structures can repress protein translation [44]. Although this suggests that the computationally intensive mRNA secondary structure evaluation may be required for designing synthetic genes, it was also reported that the helicase activity of ribosome is 
able to disrupt the secondary structures for mRNA translation [45]. Therefore, we suggest using the mRNA secondary structure analysis only as a supplementary step for the CCoptimized sequences such that no significant computational cost is added to the main $\mathrm{CCO}$ procedure.

\section{CCO tool for synthetic biology}

To further develop CCO into a software tool for designing synthetic genes, several other factors may have to be considered. From the experimental aspect, the gene optimization should take into consideration the types of restriction enzymes used for vector construction such that the restriction sites DNA motifs are avoided to prevent unnecessary cleavage of the coding sequence. In certain cases where the optimized coding sequence tends to have nucleotide repeats, additional steps may be required to avoid the repeats or inverted repeats which may lead to DNA recombination or formation of mRNA hairpin loops, respectively, that will reduce the heterologous expressivity of the target protein $[46,47]$. In addition, sequence homology may also be considered to design genes that are resistant to RNA interference such that complementary sequences of the silencing RNAs are avoided in the coding sequence [48]. Possible strategies to tackle the aforementioned issues during gene optimization have been discussed in a previous study [20].

The optimal sequences generated by $\mathrm{CCO}$ are not found in any natural organism. Thus, the CCO software tool should also consider challenges involved in the synthesis of these artificial genes. The current technology for de novo gene synthesis involves the chemical synthesis of short oligonucleotides followed by ligation- or PCRmediated assembly of the oligonucleotides to form the complete gene [49]. The way in which a long coding sequence is broken down into short oligonucleotides has to be properly designed to minimize the oligonucleotide synthesis error rate and maximize the uniformity of the oligonucleotides' annealing temperatures for efficient assembly. Several methods such as DNAWorks [50], Gene2Oligo [51] and TmPrime [52] have been proposed to these goals in oligonucleotide design optimization for gene synthesis. Although these oligonucleotide optimization methods can be performed independently from the codon optimization procedure, these two processes can be integrated to facilitate the "design-to-synthesis" workflow. As long as the current gene synthesis paradigm prevails, researchers can further explore the possibility of developing an integrated codon and oligonucleotide optimization software tool to effectively and systematically design high performance synthetic genes for protein expression.

\section{Potential applications of CCO}

The motivation behind codon optimization is usually to enhance the expression of foreign genes in expression hosts such as E. coli, P. pastoris and S.cerevisiae. In addition, codon optimization can also be used to generate synthetic designs of native genes for metabolic engineering applications. While conventional overexpression of native metabolic genes is achieved by increasing gene copy number through the introduction of plasmids, codon optimization provides an alternative approach for enhancing pathway utilization via insertion of highexpression synthetic genes of the respective metabolic enzymes into the host's genome. The latter technique can be advantageous as it obviates the metabolic burden associated with plasmid maintenance [53-55], thus allowing the cells to have more resources for growth and biochemical production.

Apart from biotechnological applications, codon optimization can also be used in biomedical research where modulation of protein expression is required to alter physiological response. For example, in the development of vaccines against viruses, one approach is to genetically manipulate the virus to obtain a "live attenuated" strain as the vaccine. Such a vaccine, when administered to the host, will elicit an immune response for the host to develop immunologic memory and specific immunity against the virus without severe disruption to the overall physiology. Some conventional methods of developing live attenuated vaccines include laboratory adaptation of virus in non-human hosts and random/sitedirected mutagenesis [56]. Since the wild-type virus is able to hijack the gene expression machinery of the host for replication, the de-optimization of viral codon usage can lead to the development of live attenuated vaccines as demonstrated in a recent study [19]. Therefore, the CCO framework developed in this study can be slightly modified to design synthetic virus consisting of more rare codons that can be used as vaccines. Specifically, we can either invert the objective function to minimize $\mathrm{CC}$ fitness or alter the target $\mathrm{CC}$ distribution during the execution of the optimization procedure to design the sequence of the attenuated virus.

\section{Conclusions}

Through novel implementations of ICO, CCO and MOCO, the high-expression genes of four microbial hosts were optimized and cross-validated to compare the performance of the optimized sequences. Amongst all the optimization approaches, $\mathrm{CCO}$ was found to generate the sequences that are most similar to the native high-expression genes, indicating a greater potential for high in vivo protein expression. Contrary to the conventional practice which adopts ICU optimization as the key element of gene design, our study suggests that $\mathrm{CC}$ fitness is a more relevant design parameter for optimizing the sequence for improved heterologous protein expression. Thus, future works to incorporate the 
optimization of CC fitness into synthetic gene design software can lead to the development of more efficient platforms for gene optimization.

\section{Methods}

Identifying highly expressed genes

Provided that highly expressed genes have evolved to adopt optimal codon patterns, information on ICU and $\mathrm{CC}$ preference of any organism can be extracted from the DNA sequences of the high-expression genes. In this sense, we used published microarray data of E. coli [57], L. lactis [58], P. pastoris [59] and S. cerevisiae [60] from various experimental conditions to identify the top $5 \%$ of genes with the highest expression value for each microbe. The ICU and CC of these genes were then extracted from their corresponding DNA coding sequences that can be obtained from publicly available genome annotations for E. coli [61], L. lactis [62], P. pastoris [63] and S. cerevisiae [64]. Each host's ICU and CC preference can be represented as the frequency of occurrence of individual codons and codon pairs found in the sequences of the highly expressed genes. These ICU and CC distributions are then be used as the targets for the respective codon optimization methods. For the evaluation of ICU and CC biasness difference between high- and low-expression genes, the low-expression genes are identified in a similar way whereby the bottom $5 \%$ of the genes with the lowest expression values are consolidated (see Additional file 1 for a list of highexpression genes).

\section{ICU and CC biasness}

To compute the significance of codon (pair) usage bias, we resort to the Pearson's chi-squared test. Based on the null hypothesis that "the ICU (CC) of highexpression genes follows the uniform/unbiased distribution", the chi-square statistic for amino acid (pair) $j$ is calculated as:

$$
\mathrm{X}_{j}^{2}=\sum_{i=1}^{n_{j}^{H}} \frac{\left(O_{i j}^{H}-E_{i j}^{H}\right)^{2}}{E_{i j}^{H}}
$$

where $E_{i j}^{H}$ and $O_{i j}^{H}$ are the expected and observed numbers of synonymous codon (pair) $i$ encoding amino acid (pair) $j$, respectively. The constant $n_{j}^{H}$ refers to the number of unique synonymous codon (pair) encoding the amino acid $j$; for example, the $n_{j}^{H}$ values for asparagine, glycine and leucine are 2, 4, and 6 respectively. The superscript " $H$ " indicates that only the high-expression genes are used to evaluate the respective values. Given the null hypothesis of unbiased codon (pair) usage, $E_{i j}^{H}$ can be calculated as $E_{i j}^{H}=\frac{N_{j}^{H}}{n_{j}^{H}}$ where $N_{j}^{H}$ refers to the total number of amino acid (pair) $j$ found in the high-expression genes. The pvalue is then evaluated by comparing the calculated $X_{j}^{2}$ against the $X^{2}$ distribution with $\left(n_{j}^{H}-1\right)$ degrees of freedom since the reduction in degrees of freedom is one due to the constraint: $\sum_{i=1}^{n_{j}^{H}} O_{i j}^{H}=N_{j}^{H}$. Using a p-value cut-off of 0.05 , we can identify the amino acid (pair) with biased ICU (CC) distribution that is significantly different from the normal distribution. This test of ICU and CC biasness will be referred to as " $X{ }^{2}$ Test 1 ". To ensure that the statistical adequacy of this chi-squared test, any amino acid (pair) with low expected occurrence (i.e. $\left.E_{i j}^{H}<5\right)$ will be omitted from this analysis as recommended in an earlier study [65]. Furthermore, chisquared test of singular amino acids (methionine and tryptophan) and amino acid pairs (pairs only consisting of methionine and/or tryptophan) are also not relevant since they are not encoded by more than one synonymous codon (pair) such that the chi-squared statistic will always be equal to 1 .

The presented Pearson's chi-squared formulation is slightly modified to determine whether the ICU (CC) is significantly different between high-expression genes and all genes in the genome. Based on the null hypothesis as "ICU (CC) of high-expression genes is the same as that of all genes in the genome", the expected number of codon (pair) $i$ in high-expression genes is modified as:

$$
\tilde{E}_{i j}^{H}=\frac{O_{i j}^{A} N_{j}^{H}}{N_{j}^{A}}
$$

where $O_{i j}^{A}$ refers the observed number of codon (pair) $i$ encoding amino acid (pair) $j$ and $N_{j}^{A}$ refers to the total number of amino acid (pair) $j$. The superscript " $A$ " indicates that all genes in the host's genome are used for evaluating the respective values. By substituting $E_{i j}^{H}$ with $\tilde{E}_{i j}^{H}$ in the expression for $X_{j}^{2}$, the chisquared statistic to test the difference in ICU (CC) distribution between high-expression genes and all genes in the host's genome can be calculated.

\section{ICU and CC fitness evaluation}

In this study, the target gene, subsequently known as the "subject", is optimized such that the final synthetic sequence design will exhibit ICU and/or CC distributions that are as similar as possible to those preferred by the host's organism. The ICU and CC fitness values can be used to quantify the degree of similarity in ICU and CC distributions between the subject and the host. Before formulating the ICU and CC fitness, we present the mathematical expression of the coding sequence and 
amino acid sequence as follows:

$$
\begin{aligned}
S_{\mathrm{A}, 1}= & \{M, R, F, P, S, I, F, \ldots, G, D, R, *\} \\
= & \left\{\tau_{i, 1}\right\}_{i=1}^{n} \\
S_{\mathrm{C}, 1}= & \{A U G, A G A, \text { UUU }, \text { CCU, UCA }, \ldots, G A C, \\
& \text { AGA,UGA }=\left\{\lambda_{i, 1}\right\}_{i=1}^{n} \\
\tau_{i, 1} \in \mathrm{A}= & \left\{\alpha^{j}\right\}_{j=1}^{21}=\{A, C, D, \ldots, W, Y, *\} \quad \forall i \\
\lambda_{i, 1} \in \mathrm{K}=\left\{\kappa^{k}\right\}_{k=1}^{64} & \\
= & \{A A A, A A C, A A G, \ldots, \text { UUG }, \text { UUU }\} \quad \forall i
\end{aligned}
$$

where $\tau_{i, 1}$ refers to the amino acid occupying the $i^{\text {th }}$ position of the amino acid sequence $S_{\mathrm{A}, 1}$ with the subscript 1 indicating the target protein; $\tau_{i, 1}$ also belongs to the set A of 21 unique amino acids $\alpha^{j}$. Similarly, $\lambda_{i, 1}$, a codon from the set $K$ of 64 unique codons $k^{k}$, represents the codon variable in the $i^{\text {th }}$ position of the target coding sequence $S_{\mathrm{C}, 1}$. It is noted that the coding sequence is express as a sequence of codons instead of nucleotides since codon usage patterns is the key concern. As codon context is another key issue to be examined, we also include the following mathematical expressions for amino acid pairs and codon pairs:

$$
\begin{aligned}
S_{\mathrm{AA}, 1}= & \{M R, R F, F P, P S, S I, \ldots, G D, D R, R *\} \\
= & \left\{\omega_{i, 1}\right\}_{i=1}^{n-1} \\
S_{\mathrm{CC}, 1}= & \{\text { AUGAGA, AGAUUU, UUUCCU }, \ldots, \\
& \text { AGAUGA }\}=\left\{\gamma_{i, 1}\right\}_{i=1}^{n-1} \\
\omega_{i, 1} \in \mathrm{B}= & \{\text { AA, AC, CA }, \ldots, W *, Y *\} \\
= & \left\{\beta^{j}\right\}_{j=1}^{420} \quad \forall i \in\{1, \ldots, n-1\} \\
\gamma_{i, 1} \in \mathrm{P}= & \{\text { AAAAAA, } ., \text { UUUUUU }\} \\
= & \left\{\rho^{k}\right\}_{k=1}^{3904} \quad \forall i \in\{1, \ldots, n-1\}
\end{aligned}
$$

By defining a function to $f$ translate codon(s) to the corresponding amino acid(s) and a concatenation function $g(a, b)$ to append the string $b$ to right of string $a$, we have the following mathematical relationships for $\tau_{i, 1}$, $\omega_{i, 1}, \lambda_{i, 1}$ and $\gamma_{i, 1}$ :

$$
\begin{aligned}
& f\left(\lambda_{i, 1}\right)=\tau_{i, 1} \\
& f\left(\gamma_{i, 1}\right)=\omega_{i, 1} \\
& g\left(\tau_{i, 1}, \tau_{(i+1), 1}\right)=\omega_{i, 1} \\
& g\left(\lambda_{i, 1}, \lambda_{(i+1), 1}\right)=\gamma_{i, 1}
\end{aligned}
$$

The ICU distribution can be defined as the frequency of each unique codon based on its total number of occurrences in the sequence(s). Based on the mathematical formulation presented hitherto, the required mathematical expressions to calculate the ICU distribution are as follows:

$$
\begin{aligned}
& \theta_{\mathrm{A}, 1}^{\mathrm{j}}=\sum_{i=1}^{n} 1\left\{\tau_{i, 1}=\alpha^{\mathrm{j}}\right\} \quad \forall j \in\{1,2, \ldots, 21\} \\
& \theta_{\mathrm{C}, 1}^{\mathrm{k}}=\sum_{i=1}^{n} 1\left\{\lambda_{i, 1}=\kappa^{\mathrm{k}}\right\} \quad \forall k \in\{1,2, \ldots, 64\} \\
& \theta_{\mathrm{A}, 0}^{\mathrm{j}}=\sum_{i=1}^{n^{\prime}} 1\left\{\tau_{i, 0}=\alpha^{\mathrm{j}}\right\} \quad \forall j \in\{1,2, \ldots, 21\} \\
& \theta_{\mathrm{C}, 0}^{\mathrm{k}}=\sum_{i=1}^{n^{\prime}} 1\left\{\lambda_{i, 0}=\kappa^{\mathrm{k}}\right\} \quad \forall k \in\{1,2, \ldots, 64\} \\
& p_{0}^{k}=\frac{\theta_{\mathrm{C}, 0}^{21}\left[\theta_{\mathrm{A}, 0}^{j} \times 1\left\{\alpha^{j}=f\left(\kappa^{k}\right)\right\}\right]}{\sum_{j=1}^{k}} \quad \forall k \in\{1,2, \ldots, 64\} \\
& p_{1}^{k}=\frac{\theta_{\mathrm{C}, 1}^{k}}{\sum_{j=1}^{21}\left[\theta_{\mathrm{A}, 1}^{j} \times 1\left\{\alpha^{j}=f\left(\kappa^{k}\right)\right\}\right]} \\
& \theta_{\mathrm{C}, 0}^{k}=\sum_{i=1}^{n^{\prime}} 1\left\{\lambda_{i, 0}=\kappa^{k}\right\} \quad \forall k \in\{1,2, \ldots, 64\} \\
& \theta_{\mathrm{C}, 0}^{k}=\sum_{i=1}^{n^{\prime}} 1\left\{\lambda_{i, 0}=\kappa^{k}\right\} \quad \forall k \in\{1,2, \ldots, 64\}
\end{aligned}
$$

where $1\{\bullet\}$ is an indicator function such that $1\{x\}=\left\{\begin{array}{ll}1 & \text { if } x \text { is true } \\ 0 & \text { otherwise }\end{array}\right.$.

The count variables $\theta_{\mathrm{AA}}^{j}$ and $\theta_{\mathrm{C}}^{k}$ refer to the numbers of occurrences of amino acid $j$ and codon $k$, respectively, found in the host's (indicated by subscript " 0 ") or subject's (indicated by subscript "1") sequence(s), while $p^{k}$ represents the frequency of occurrence of codon $k$. Accordingly, the ICU fitness can be expressed as:

$$
\Psi_{\mathrm{ICU}}=-\frac{\sum_{k=1}^{64}\left|p_{0}^{k}-p_{1}^{k}\right|}{64}
$$

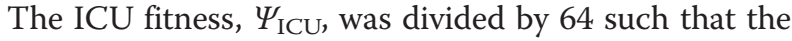
numerical value will reflect the average fitness of all codons. In a similar way, if we denote the frequency of occurrence of codon pair $k$ as $q^{k}$, the CC fitness can be calculated as: 


$$
\begin{array}{cc}
q_{0}^{k}=\frac{\theta_{\mathrm{CC}, 0}^{k}}{\sum_{j=1}^{420}\left[\theta_{\mathrm{AA}, 0}^{j} \times 1\left\{\beta^{j}=f\left(\rho^{k}\right)\right\}\right]} & \forall k \in\{1,2, \ldots, 3904\} \\
q_{1}^{k}=\frac{\theta_{\mathrm{CC}, 1}^{k}}{\sum_{j=1}^{420}\left[\theta_{\mathrm{AA}, 1}^{j} \times 1\left\{\beta^{j}=f\left(\rho^{k}\right)\right\}\right]} & \forall k \in\{1,2, \ldots, 3904\}
\end{array}
$$

$$
\Psi_{\mathrm{CC}}=-\frac{\sum_{k=1}^{3904}\left|q_{0}^{k}-q_{1}^{k}\right|}{3904}
$$

(See Additional file 3 for further details of the mathematical formulation).

\section{Additional files}

Additional file 1: List of Sequences. Excel file contains DNA sequences of wild-type high- and low-expression genes; the optimized genes generated by the in silico leave-one-out cross validation are also included.

Additional file 2: Codon optimization of another set of highexpression genes in E. coli. ICO, CCO and MOCO were carried out using the set of high-expression genes reported in an earlier study [33] to evaluate the relative performance of the methods.

Additional file 3: Formulation of codon optimization methods. Detailed mathematical formulation of ICO, CCO and MOCO.

\section{Competing interests}

The authors declare that they have no competing interests.

\section{Authors' contributions}

BKSC and DL conceived the codon optimization idea. BKSC developed the algorithm, performed the computational simulations and drafted the manuscript. DL revised the manuscript. Both authors read and approved the final manuscript.

\section{Acknowledgements}

This work was supported by the National University of Singapore, Biomedical Research Council of A*STAR (Agency for Science, Technology and Research), Singapore and a grant from the Next-Generation BioGreen 21 Program (SSAC, No. PJ008184), Rural Development Administration, Republic of Korea. We would like to thank Dr. Jungoh Ahn and the anonymous reviewers for the invaluable suggestions and feedbacks.

\section{Author details}

${ }^{1}$ Department of Chemical and Biomolecular Engineering, National University of Singapore, 4 Engineering Drive 4, Singapore 117576, Singapore. ${ }^{2}$ NUS Graduate School for Integrative Sciences and Engineering, National University of Singapore, 28 Medical Drive, \#05-01, Singapore 117456, Singapore. ${ }^{3}$ Bioprocessing Technology Institute, Agency for Science, Technology and Research (A*STAR), 20 Biopolis Way, \#06-01 Centros, Singapore 138668, Singapore.

Received: 5 June 2012 Accepted: 11 October 2012 Published: 20 October 2012

\section{References}

1. Nandagopal N, Elowitz MB: Synthetic biology: integrated gene circuits. Science 2011, 333:1244-1248.
2. Gibson DG, Glass Jl, Lartigue C, Noskov VN, Chuang RY, Algire MA, Benders GA, Montague MG, Ma L, Moodie MM, et al: Creation of a bacterial cell controlled by a chemically synthesized genome. Science 2010, 329:52-56.

3. Nielsen J: Metabolic engineering. Appl Microbiol Biotechnol 2001, 55:263-283.

4. Lee SY, Lee DY, Kim TY: Systems biotechnology for strain improvement. Trends Biotechnol 2005, 23:349-358.

5. Grantham R, Gautier C, Gouy M, Mercier R, Pave A: Codon catalog usage and the genome hypothesis. Nucleic Acids Res 1980, 8:r49-r62.

6. Hershberg R, Petrov DA: Selection on codon bias. Annu Rev Genet 2008, 42:287-299.

7. Gouy M, Gautier C: Codon usage in bacteria: correlation with gene expressivity. Nucleic Acids Res 1982, 10:7055-7074.

8. Duret L, Mouchiroud D: Expression pattern and, surprisingly, gene length shape codon usage in Caenorhabditis, Drosophila, and Arabidopsis. Proc Natl Acad Sci U S A 1999, 96:4482-4487.

9. Gustafsson C, Govindarajan S, Minshull J: Codon bias and heterologous protein expression. Trends Biotechnol 2004, 22:346-353.

10. Fuglsang A: Codon optimizer: a freeware tool for codon optimization. Protein Expr Purif 2003, 31:247-249.

11. Villalobos A, Ness JE, Gustafsson C, Minshull J, Govindarajan S: Gene Designer: a synthetic biology tool for constructing artificial DNA segments. BMC Bioinforma 2006, 7:285.

12. Puigbo P, Guzman E, Romeu A, Garcia-Vallve S: OPTIMIZER: a web server for optimizing the codon usage of DNA sequences. Nucleic Acids Res 2007, 35:W126-W131.

13. Grote A, Hiller K, Scheer M, Munch R, Nortemann B, Hempel DC, Jahn D: JCat: a novel tool to adapt codon usage of a target gene to its potential expression host. Nucleic Acids Res 2005, 33:W526-W531.

14. Munch R, Hiller K, Barg H, Heldt D, Linz S, Wingender E, Jahn D: PRODORIC: prokaryotic database of gene regulation. Nucleic Acids Res 2003, 31:266-269.

15. Yarus M, Folley LS: Sense codons are found in specific contexts. $J$ Mol Biol 1985, 182:529-540.

16. Tats A, Tenson T, Remm M: Preferred and avoided codon pairs in three domains of life. BMC Genomics 2008, 9:463.

17. Gutman GA, Hatfield GW: Nonrandom utilization of codon pairs in Escherichia coli. Proc Natl Acad Sci U S A 1989, 86:3699-3703.

18. Smith D, Yarus M: tRNA-tRNA interactions within cellular ribosomes. Proc Natl Acad Sci U S A 1989, 86:4397-4401.

19. Coleman JR, Papamichail D, Skiena S, Futcher B, Wimmer E, Mueller S: Virus attenuation by genome-scale changes in codon pair bias. Science 2008, 320:1784-1787.

20. Raab D, Graf M, Notka F, Schodl T, Wagner R: The GeneOptimizer Algorithm: using a sliding window approach to cope with the vast sequence space in multiparameter DNA sequence optimization. Syst Synth Biol 2010, 4:215-225.

21. Hatfield GW, Roth DA: Optimizing scaleup yield for protein production Computationally Optimized DNA Assembly (CODA) and Translation Engineering. Biotechnol Annu Rev 2007, 13:27-42.

22. Wildt S, Gerngross TU: The humanization of N-glycosylation pathways in yeast. Nat Rev Microbiol 2005, 3:119-128.

23. Morello E, Bermudez-Humaran LG, Llull D, Sole V, Miraglio N, Langella P, Poquet I: Lactococcus lactis, an efficient cell factory for recombinant protein production and secretion. J Mol Microbiol Biotechnol 2008, 14:48-58.

24. Chung BK, Lee DY: Flux-sum analysis: a metabolite-centric approach for understanding the metabolic network. BMC Syst Biol 2009, 3:117.

25. Atamtürk A, Savelsbergh M: Integer-Programming Software Systems. Ann Oper Res 2005, 140:67-124.

26. Sandhu KS, Pandey S, Maiti S, Pillai B: GASCO: genetic algorithm simulation for codon optimization. In Silico Biol 2008, 8:187-192.

27. Cai Y, Sun J, Wang J, Ding Y, Liao X, Xu W: QPSOBT: One codon usage optimization software for protein heterologous expression. $J$ Bioinformatics Seq Anal 2010, 2:25-29.

28. Applegate DL: The traveling salesman problem: a computational study. Princeton University Press; 2006

29. Goldberg DE: Genetic algorithms in search, optimization, and machine learning. Addison-Wesley Pub. Co.; 1989

30. Deb K, Pratap A, Agarwal S, Meyarivan T: A fast and elitist multiobjective genetic algorithm: NSGA-II. IEEE Trans Evo Comp 2002, 6:182-197. 
31. Zouridis $H$, Hatzimanikatis $\mathrm{V}$ : A model for protein translation: polysome self-organization leads to maximum protein synthesis rates. Biophys $J$ 2007, 92:717-730.

32. Zouridis $\mathrm{H}$, Hatzimanikatis $\mathrm{V}$ : Effects of codon distributions and tRNA competition on protein translation. Biophys J 2008, 95:1018-1033.

33. Sharp PM, Li WH: Codon usage in regulatory genes in Escherichia coli does not reflect selection for 'rare' codons. Nucleic Acids Res 1986, 14:7737-7749.

34. Sharp PM, Li WH: The codon adaptation index-a measure of directional synonymous codon usage bias, and its potential applications. Nucleic Acids Res 1987, 15:1281-1295

35. Nakamura $Y$, Gojobori T, Ikemura T: Codon usage tabulated from international DNA sequence databases: status for the year 2000. Nucleic Acids Res 2000, 28:292.

36. Kane JF: Effects of rare codon clusters on high-level expression of heterologous proteins in Escherichia coli. Curr Opin Biotechnol 1995, 6:494-500.

37. Kurland C, Gallant J: Errors of heterologous protein expression. Curr Opin Biotechnol 1996, 7:489-493.

38. Stoletzki N, Eyre-Walker A: Synonymous codon usage in Escherichia coli: selection for translational accuracy. Mol Biol Evol 2007, 24:374-381.

39. Sorensen MA, Kurland CG, Pedersen S: Codon usage determines translation rate in Escherichia coli. J Mol Biol 1989, 207:365-377.

40. Racle J, Overney J, Hatzimanikatis V: A computational framework for the design of optimal protein synthesis. Biotechnol Bioeng 2012

41. Heinrich $R$, Rapoport TA: Mathematical modelling of translation of mRNA in eucaryotes; steady state, time-dependent processes and application to reticulocytes. J Theor Biol 1980, 86:279-313.

42. Bulmer M: The selection-mutation-drift theory of synonymous codon usage. Genetics 1991, 129:897-907.

43. Kozak M: Initiation of translation in prokaryotes and eukaryotes. Gene 1999, 234:187-208

44. Kozak M: Regulation of translation via mRNA structure in prokaryotes and eukaryotes. Gene 2005, 361:13-37.

45. Takyar S, Hickerson RP, Noller HF: mRNA helicase activity of the ribosome. Cell 2005, 120:49-58.

46. Bzymek M, Lovett ST: Instability of repetitive DNA sequences: the role of replication in multiple mechanisms. Proc Natl Acad Sci U S A 2001, 98:8319-8325

47. Kudla G, Murray AW, Tollervey D, Plotkin JB: Coding-sequence determinants of gene expression in Escherichia coli. Science 2009, 324:255-258.

48. Kim DH, Rossi JJ: Coupling of RNAi-mediated target downregulation with gene replacement. Antisense Nucleic Acid Drug Dev 2003, 13:151-155.

49. Hughes RA, Miklos AE, Ellington AD: Gene synthesis: methods and applications. Methods Enzymol 2011, 498:277-309.

50. Hoover DM, Lubkowski J: DNAWorks: an automated method for designing oligonucleotides for PCR-based gene synthesis. Nucleic Acids Res 2002, 30:e43.

51. Rouillard JM, Lee W, Truan G, Gao X, Zhou X, Gulari E: Gene2Oligo: oligonucleotide design for in vitro gene synthesis. Nucleic Acids Res 2004, 32:W176-180.

52. Bode M, Khor S, Ye H, Li MH, Ying JY: TmPrime: fast, flexible oligonucleotide design software for gene synthesis. Nucleic Acids Res 2009, 37:W214-221.

53. Corchero $J$ L, Villaverde A: Plasmid maintenance in Escherichia coli recombinant cultures is dramatically, steadily, and specifically influenced by features of the encoded proteins. Biotechnol Bioeng 1998, 58:625-632.

54. Diaz Ricci JC, Hernandez ME: Plasmid effects on Escherichia coli metabolism. Crit Rev Biotechnol 2000, 20:79-108.

55. Ow DS, Lee DY, Yap MG, Oh SK: Identification of cellular objective for elucidating the physiological state of plasmid-bearing Escherichia coli using genome-scale in silico analysis. Biotechnol Prog 2009, 25:61-67.

56. Wareing MD, Tannock GA: Live attenuated vaccines against influenza; an historical review. Vaccine 2001, 19:3320-3330.

57. Faith JJ, Hayete B, Thaden JT, Mogno I, Wierzbowski J, Cottarel G, Kasif S, Collins JJ, Gardner TS: Large-scale mapping and validation of Escherichia coli transcriptional regulation from a compendium of expression profiles. PLOS Biol 2007, 5:e8.
58. Dressaire $C$, Redon $E$, Milhem $H$, Besse $P$, Loubiere $P$, Cocaign-Bousquet $M$ : Growth rate regulated genes and their wide involvement in the Lactococcus lactis stress responses. BMC Genomics 2008, 9:343.

59. Vijay Kumar N, Rangarajan PN: Catabolite repression of phosphoenolpyruvate carboxykinase by a zinc finger protein under biotin- and pyruvate carboxylase-deficient conditions in Pichia pastoris. Microbiology 2011, 157:3361-3369.

60. Knijnenburg TA, Daran JM, van den Broek MA, Daran-Lapujade PA, de Winde JH, Pronk JT, Reinders MJ, Wessels LF: Combinatorial effects of environmental parameters on transcriptional regulation in Saccharomyces cerevisiae: a quantitative analysis of a compendium of chemostat-based transcriptome data. BMC Genomics 2009, 10:53.

61. Riley M, Abe T, Arnaud MB, Berlyn MK, Blattner FR, Chaudhuri RR, Glasner JD, Horiuchi T, Keseler IM, Kosuge T, et al: Escherichia coli K-12: a cooperatively developed annotation snapshot-2005. Nucleic Acids Res 2006, 34:1-9.

62. Bolotin A, Wincker $P$, Mauger S, Jaillon O, Malarme K, Weissenbach J, Ehrlich $\mathrm{SD}$, Sorokin A: The complete genome sequence of the lactic acid bacterium Lactococcus lactis ssp. lactis IL1403. Genome Res 2001, 11:731-753.

63. De Schutter K, Lin YC, Tiels P, Van Hecke A, Glinka S, Weber-Lehmann J, Rouze P, Van de Peer Y, Callewaert N: Genome sequence of the recombinant protein production host Pichia pastoris. Nat Biotechnol 2009, 27:561-566.

64. Hong EL, Balakrishnan R, Dong Q, Christie KR, Park J, Binkley G, Costanzo MC, Dwight SS, Engel SR, Fisk DG, et al: Gene Ontology annotations at SGD: new data sources and annotation methods. Nucleic Acids Res 2008, 36:D577-581.

65. Cochran WG: Some Methods for Strengthening the Common X2 Tests. Biometrics 1954, 10:417-451.

doi:10.1186/1752-0509-6-134

Cite this article as: Chung and Lee: Computational codon optimization of synthetic gene for protein expression. BMC Systems Biology 2012 6:134.

\section{Submit your next manuscript to BioMed Central and take full advantage of:}

- Convenient online submission

- Thorough peer review

- No space constraints or color figure charges

- Immediate publication on acceptance

- Inclusion in PubMed, CAS, Scopus and Google Scholar

- Research which is freely available for redistribution 